\title{
The Student as Philosopher-Scientist: Dewey's Conception of Scientific Explanation In Science Education
}

\author{
Mark D. Tschaepe
}

\section{Abstract}

Dewey's conception of scientific explanation, which has been neglected by both philosophers of science and philosophers of education, facilitates overcoming the seeming divide between teaching a highly technical and specialized subject matter and encouraging students to successfully engage in the experience of being philosopher-scientists. By analyzing Dewey's philosophy of science as it pertains to science education, we gain the insight that scientific explanation is a tool that may be used by students and supported by faculty to facilitate scientific investigation that is philosophical. Here I present Dewey's conception of scientific explanation as it relates to science education by providing an overview of this conception as it relates to the student, followed by Dewey's ideas with specific regard for science education, as well as an example illustrating how scientific explanation is utilized as a self-empowering, philosophical tool within the context of science education.

There is no question that the work of John Dewey has been invaluable with regard to theories of education. What has too often been neglected, however, is Dewey's work on the philosophy of science as it pertains specifically to science education. ${ }^{1}$ Although educators might well concede that children should be encouraged to be "philosophical" within the arts or humanities, most neglect or fail to heed Dewey's insights concerning the child as philosopher-scientist within the science classroom. Dewey recognized that children were too often taught "science" while not learning 
"the scientific way of treating the familiar material of ordinary experience" (MW 9: 228). The view of science as a nonphilosophical, highly technical subject that is somehow fundamentally separated from other subjects remains a problem within education. Recently, for example, Victor Pollak criticized science education on this score, indicating the need for reconciliation between the "fundamentally antiauthoritarian spirit of science"-what Dewey considered the scientific, reconstructive manner of thinking-and "education, with its built-in tendency to be authoritarian" (Pollak, 513). In what follows, I argue that Dewey's conception of scientific explanation, which has often been neglected by both philosophers of science and philosophers of education, can assist us in overcoming the divide between teaching a highly technical and specialized subject matter and encouraging students to successfully engage in "the scientific way of treating the familiar material of ordinary experience" (MW 9: 228). When students engage in scientific explanation as a tool that facilitates learning how to be scientific, rather than memorizing scientific explanations as a series of facts, they can become philosopher-scientists.

Below, I present Dewey's conception of scientific explanation as it relates to scientific education by first providing an overview of this conception in relation to the individual student. This is followed by more pointed discussion of Dewey's thinking regarding science education, as well as examples illustrating how scientific explanation, as he sees it, should be utilized as a self-empowering philosophical tool within the context of science education. Accordingly, Dewey's conception of scientific explanation is connected with scientific education in a way that provides a basis for understanding how Dewey advocated an approach to education that fosters the child as philosopher-scientist, especially within the context of the science classroom.

As with so many concepts with which Dewey worked, his writing on this topic is infused with a very particular idea of explanation that is wedded to other concepts in his work and rooted in his theory of inquiry. The functional definition of explanation he supplies refers to "bringing a given set of cases into relation with sets that are of a different kind with respect to qualitative considerations so that free and systematic inference is possible" (LW 14: 24n24). However, this definition does not provide an adequate idea of Dewey's conception of scientific explanation, especially in the realm of science education. The following, then, is an analysis of Dewey's conception of scientific explanation that expands upon his very general functional definition.

For Dewey, scientific explanation is a particular kind of operation used in solving problems. Explanation in the context of education is, like any process in which students participate, born from transactions with the world of which students are a part. Most of students' transactions with the world are unproblematic; experiences occur and are undergone and accepted without question. However, when problems - caused by perturbations in the usually calm landscape of experiencedo occur, students are led to inquire into those problems in order to solve them so that experience will again be unproblematic and new understandings and abilities 
can be acquired. Inquiry is the activity by which initial experience-that which is uncontrolled and indeterminate-is transformed via the interrelated processes of analysis and synthesis. Analysis is the process of discrimination by which experience is discriminated or divided into particular objects of experience. Synthesis is the process of identification wherein the objects of experience are unified as a whole (LW 8: 275). For instance, in attempting to understand the sense of hearing, we might analyze the ear into its various parts and the operations those parts perform. Once we have divided the ear in this manner, we might then (re)synthesize those parts and operations in order to understand how they function together to facilitate the sense of hearing.

When problems occur, inquiry calls for observation of the facts at hand. Students discern objects of knowledge through the process of inquiry; as Dewey says, "objects of knowledge are not given to us defined, classified, and labeled, ready for labels and pigeonholes" (LW 1: 170). In order to discern objects of knowledge which students deal with in the process of inquiry, experience is analyzed as data-discernible material of experience that is purposefully divided into distinct units so that how or why a problem has occurred might be understood. To return to our previous example, imagine that students experience perturbation over the nature of hearing-what makes it possible and how it works-after encountering and wrestling with the popular Zen koan, "If a tree falls in the woods and no one is there to hear it, does it really make a sound?" After identifying the source of their perturbation (the problem or questions to be answered) and hypothesizing possible resolutions through close examination of the ear, the analytic phase of inquiry might involve the division of the ear into the outer, middle, and inner ear. In the attendant process of synthesis, students utilize this data to formulate what Dewey calls ideas, suggestions for further inquiry or possible resolutions to the problem identified through the experienced perturbation (LW 8: 197-98). With regard to hearing, the analysis of the bones of the middle ear (malleus, incus, and stapes) could be utilized in the synthesis of these parts and their functions into the idea of the transmission of sound vibrations through material. Between the analysis of experience into data and the synthesis of data into ideas, students search for solutions to the problem by altering experience in order to test what particular effects are derived from what particular causes.

The alteration of experience through inquiry brings about a type of experience distinct from so-called "primary" experience: what Dewey refers to as secondary or reflective experience. Secondary experience is experience that has been systematically analyzed and synthesized, and by which students attempt to gain an understanding of primary experience. Through the analytic function of secondary experience, scientific objects are applied to the problem identified within primary experience; scientific objects help students explain primary experience (LW 1: 15-16). In turn, the explanations generated via secondary experience are tested by whether or not they are warranted with regard to students' ability to navigate through situa- 
tions within primary experience. In the reconstruction of primary experience into secondary experience, students engage in the process of locating and demarcating causes of the effects the students are attempting to explain.

An example of a primary experience in this context might be hearing a specific sound, such as that of knocking on wood. A causal question, informed by the previous inquiry, might then arise, "How does the sound get from out here [indicating the source of the sound] into your head?" Through the process of analysis, including the location and demarcation of causes and effects concerning the transport of sound in hearing, the students begin forming an explanation, which is part of the their secondary experience. This means that the experience of inquiry is guided by the students as they engage in the construction of explanations pertaining to their primary experience. ${ }^{2}$

According to Dewey, the search for "efficient causes" is the aim of scientific practice; this is always "a search for those relations upon which the occurrence of real qualities and values depends, by means of which we can regulate their occurrence" (LW 4: 83). By varying the conditions through which various experiences occur, that is, through the process of intervention, students attempt to locate the source of the perturbation in their experience so that they can generate an understanding of the cause of the perturbation (the problem), as well as possible means of removing or diminishing it. In order to explain what is occurring in their experience, students must submit this experience to experimentation and the formulation of hypotheses. As Dewey claims, students must "produce the cases" (LW 12: 275). Through the repeated and varied production of cases, along with a fallibilist sensibility regarding preconceived notions about their experience, students engage in what Dewey refers to as the process of philosophical reconstruction.

Philosophical reconstruction, for Dewey, is the utilization of experimentation within inquiry. Students reconstruct when they regard their habitual approaches to experience - their past knowledge, beliefs, and assumptions that collectively predispose them to act in specific ways-as fallible. This process of evaluation and the implementation of experimental methods signifies the transition from naïe empiricism and mythic approaches regarding causality-the bare imposition of absolutistic concepts based upon singular experiences - to probabilistic thinking. Reconstruction is thus based upon students' transactions with the world, and these ultimately supply them with the materials for inquiry.

The process of reconstruction entails regarding intelligence-inquiry into experience and action-as purposeful and re-creative with regard to experience (MW 12: 108). In this process, students attempt to reconstruct the particular into the general-from token situations (i.e., experimental cases) to a type of situation-in order to formulate an explanation that may be used as a tool for similar situations in the future. Generalization has two distinct forms within the process of scientific explanation: that of classification of kinds (e.g., taxonomical or ontological categories of inclusion) and that of hypotheses and theories, or universal if- 
then propositions (LW 12: 422). In the case of answering the question of how sound moves from one location "outside" of the head to somewhere "inside" of the head, the student engages in both of these forms of generalization. Classification occurs in conceptualizing sound and hearing ("What is sound? What is sound like? What, exactly, does it mean to 'hear something'?"). Hypothesis-generation and theorizing occur in the process of addressing the problem at hand ("If the sound is generated in water, but my head is not underwater, does this change how I hear it? If I plug my ears, then I will not hear the sound, or I will only hear the sound faintly. If sound is a material thing, then it must have some kind of substance.") This process of generalization is not absolute, final or dogmatic; it is a continual process of identification and hypothesis-formation that is "to be worked out in practice, and to be rejected and expanded as [explanations] fail or succeed in giving our present experience the guidance it requires" (MW 12: 135). With regard to scientific explanation, reconstruction involves the attempt to formulate causal relationships and their corresponding events so as to understand and possibly alter (for the better) particular experiences. As Dewey says, students explain these causal relationships and their corresponding events by drawing upon the inferred similarity of sets of experimental cases with qualitatively different sets of cases. Explaining scientifically thus guides understanding and action, based upon experimental inquiries, with regard to causing or inhibiting a specific type of effect.

In accordance with the above, a general definition for Dewey's concept of explanation is as follows: $E$ is an explanation if $E$ provides a solution or guide to a solution for problem or question $Q$ with specific regard to an individual's or group's inquiry into a particular experience or series of experiences. This experience or series of experiences is analyzed into data, through which scientific objects are reconstructed. A scientific explanation will be based upon a series of experiments and the hypotheses that direct these experiments. The experiments are then generalized into secondary experience-conceptual language - that is applied to primary experience. The warranted assertibility (or "truth") of the scientific explanation within an educational context is based upon the combination of the real constraints and possibilities afforded by the engagement of students with their environment, the particular background considerations of the students (e.g., knowledge, habits, beliefs, assumptions) and the goals or concerns of the students in relation to $Q$ that led to explanation seeking.

Dewey's conception of scientific explanation fits seamlessly with his suggestions for science education. These suggestions entail moving away from the mere dissemination of scientific knowledge-"knowing that"-as an object for student consumption (what education theorist Richard Duschl has aptly called "final form science" [69]). Instead, Dewey advocates that students "follow . . . the methods by which scientific men have reached their perfected knowledge." He therefore stresses that students benefit from engaging in the same types of processes in which scientists engage, recognizing that these processes entail the "methods of observation, 
reflection, and testing" that make up scientific practice (MW 9: 228). This recommendation is in line with Victor Pollak's recognition "that an essential feature of science education is for the learner to participate in [the processes of scientific investigation and organization]" (515). Importantly too, studies of practices in science education that stress students engaging in the processes of inquiry in which scientists engage also provide evidence that these practices lead to both students and teachers having improved attitudes regarding science (Kyle, Bonnstetter, and Gadsen). And analysis of educational research clearly indicates that students actually do learn more efficiently and effectively as active participants, rather than as passive recipients (Lave). As Reiser, Berland, and Kenyon state, "If we expect students to learn that the scientific community builds knowledge by constructing explanations and arguments, then they must experience using these practices to address questions they have identified" (9). This is precisely the idea Dewey conveys with regard to science education.

Dewey also recognizes the need for allowing students to make mistakes when engaged in scientific inquiry. As he states, "overzeal to select materials and appliances which forbid a chance for mistakes to occur, restricts initiative, reduces judgment to a minimum, and compels the use of methods which are so remote from the complex situations of life that the power gained is of little availability" (MW 9: 205). The greatest danger when overprotective attempts are made to control students' learning how to think scientifically is that science becomes isolated "from significant experience" (228). When students are encouraged to locate and engage with problems in a manner that involves analysis, synthesis, and philosophical reconstruction - that is, the process of scientific explanation-they are engaging in the practice of being philosopher-scientists, discovering the antiauthoritarianism and strength of abstract thinking as it pertains to science.

One possible criticism of this approach is that the students might make mistakes that go uncorrected, that is, they might construct and internalize aberrant explanations that the teacher knows are incorrect. However, as Reiser et al. indicate in their analysis of an explanation-based framework for science education, "developing explanatory accounts includes not only construction but also comparison and critique. Attempts to construct new explanations typically require elements of argumentation to support and challenge potential explanations" (9). In other words, student explanations are not accepted carte blanche, especially by other students. Rather, explanations are proposals for solving problems that are recognized through the process of inquiry, and therefore those explanations are examined through the processes of experimentation and argumentation. So, in the example concerning hearing, a student could put forth an explanation that entailed small leprechauns carrying pieces of what we call sound from the source of the noise to our brains, but this explanation would be dismissed fairly quickly through the process of argumentation. This same type of process has been recorded by Nadia Kennedy with regard to mathematical inquiry. The particular example she provides is that of stu- 
dents attempting to explain, and constructing arguments concerning, the concept of infinity (Kennedy). These explanations are not dismissed by the teacher or some figure of authority, but through the processes in which the students engage together.

Engaging in the practice of being philosopher-scientists, especially with regard to the formation and utilization of scientific explanation, entails the creation of abstract concepts. According to Dewey, this is one of the most useful functions of science. The scientist, as well as the student as philosopher-scientist, develops abstractions "like a manufacturer of tools who does not know who will use them or when" (MW 9: 234). Rather than merely being told that such-and-such scientific explanations hold in such-and-such situations, the student as philosopher-scientist creates scientific explanations through an experimental process that gradually leads to the creation of abstractions that are to be tested against experience and held at ready for future problem solving. In the case of explaining the sensation of hearing, students begin by constructing explanations pertaining to a particular instance of hearing, but this is simply an initial step in creating abstractions that pertain to many instances of hearing, as well as other sensory experiences. As Dewey states, "scientific formulation supplies one with tools for constructing new experiences with transformed meanings" (235-36). This engagement in the process of scientific explanation is not only relevant to science education, as if science education was divided from other educational processes. Scientific explanation, especially in the context of science education, is also philosophical.

The creation and confirmation of abstract concepts derived from observation, experimentation, and scientific explanation provide tools for future use, especially with regard to future problematic situations. This process falls under the aegis of what Dewey defines as philosophy: "an idea of what is possible, not a record of accomplished fact" (MW 9: 336). The value of philosophy is shared with the value of scientific explanation. Engaging in the philosophical process of scientific explanation within the context of science education aids "in defining difficulties and suggesting methods for dealing with them" (336). Note that this process also contributes to Dewey's technical concept of education: "reconstruction or reorganization of experience which adds to the meaning of experience, and which increases ability to direct the course of subsequent experience" (82-83). The student thus engages in philosophical thinking, not within some realm partitioned off from the rest of education, but within all realms of education, including science education.

Another possible objection to advocating that science education entail students becoming philosopher-scientists, especially at the elementary school level, is that children, developmentally, could not possibly engage in such complex endeavors as analysis, synthesis, and reconstruction of problematic phenomena. Looking at a hypothetical example, as well as other documented examples, this seems to be a red herring. Consider, for instance, if elementary school students were shown owl pellets and then freely inquired, through their own experienced perturbation, "What are these?" The teacher might then ask, "How can we determine that?", and then once the students begin to discern what the owl pellets are: "What kinds of 
food do owls eat?" and "How would you determine this?" It does not seem difficult to concede that most children whose interest was piqued in this way would want to investigate these problematic questions, formulating their own explanations, as scientists do, based upon observation and rudimentary experiment. In fact, children do not necessarily need to be presented with specific objects of analysis in order to engage in the process of being philosopher-scientists: often, by simply not limiting them from imaginative investigation of the world, we encourage children's scientific engagement with it. An example of such engagement can be seen in Lowell Getz who, as a child in rural Illinois, came to the hypothesis that prairie voles were monogamous through the process of exploring farm fields and becoming entranced by finding male and female prairie voles consistently together. This childhood engagement led to a career as a zoologist and the founding of studies in prairie vole behavior that eventually led to major strides in neuroendocrinology and research on hormones. (In fact, Getz published a paper in 1974, "Fun in Research," that is consistent with Dewey's conceptions of science and education). Without artificial limitations imposed on him, Getz was able to freely engage in the actual process of scientific inquiry and explanation.

Such active inquiry is also advocated by Brooksfield and Preshill in a more directive form by proposing questions that ask for clarification, that are hypothetical, that address cause-and-effect relationships, and that entail analysis and synthesis of problems (Brooksfield and Preshill). Evidence indicates that children, in fact, do benefit from these types of open-ended scientific inquiry. In Developing More Curious Minds, for instance, John Barell provides numerous examples of successful science education that utilizes Dewey's principles of reconstruction and provides platforms for students to be philosopher-scientists. What such examples indicate is that students, when provided with a context for open-ended inquiry, successfully engage in tasks, such as direct observation and synthesis of prior knowledge, which are considered conceptual standards for scientific education from ninth through twelfth grade (Barell, 30).

One of the most outstanding examples Barell provides is that of an eighthgrade student, Amber Overstreet, who had observed tropical fish living in a fresh water pond 157 miles from where they would naturally live. Through her own interest and active inquiry, Overstreet questioned how the fish survived. She systematically investigated water temperatures, which led her to discover a warm water spring that provided the fish with a survivable, natural habitat. From this, she formulated a scientific explanation stating that, given the constant temperature of water in a given area, the tropical fish would survive (Barell, 33-34). This is one example, among many others, that Barell provides indicating student success in generating scientific explanation when those students are provided with the opportunity to be philosopher-scientists.

Two researchers in science education, William A. Sandoval and Brian J. Reiser, have created a computer program for science education, the ExplanationConstructor, based upon explanation-driven inquiry. They designed it, in part, as a way to reject 
the notion of science education as a process "where theoretical ideas are presented as incontrovertible facts" (Sandoval and Reiser, 346). Accordingly, the program is a tool that facilitates students engaging in the philosophical-scientific practice of generating causal explanations and reconstructing data in order to effectively argue for those explanations. In their initial studies of the ExplanationConstructor, Sandoval and Reiser found that it functioned as an epistemic tool that aided in the construction of explanations and the analysis of data. Their goal in these studies was not to evaluate the effectiveness of their program (366). What their findings do indicate, though, is that students are, in fact, able to engage in complex forms of scientific explanation generation and reconstruction. Science education is therefore not necessarily restricted to an authority-based or lecture-based form of delivery, especially regarding the subject of causal explanations.

Another possible retort to the idea of the student as philosopher-scientist could be that this is simply not how children learn, that they require more directive instruction and formal structures. However, analysis of how the brain functions and engages in what is often referred to as "higher-order thinking" indicates that the philosophical reconstructive activities that Dewey proffered, such as the formulation of scientific explanations and hypothesis generation, provide a successful foundation from which students become critical thinkers who are able to address and solve complex problems (Sousa). With regard to brain development, moreover, David A. Sousa notes the importance of self-concept, which is the student's sense of identity, in this case, with regard to a particular subject matter (57-60). This sense of identity is an important aspect of science education that is arguably strengthened by approaching the child as philosopher-scientist. Through active, positive investment in the subject matter, the student becomes emotionally receptive to the problems encountered, the processes by which those problems are addressed, and the solutions to those problems. The latter is positively reinforced and contributes to the student's self-concept because the solutions are created through the process of scientific explanation generated by the student. This approach to science education also contributes to memory retention because of the positive association with the subject matter provided by the student's personal investment. ${ }^{3}$

Unless instructed to do otherwise, children do not hesitate to engage in what Pollak has called the "being mode" of learning, wherein they take an "interest in exploring and in playing with various facets of the subject or activity at hand" (Pollak, 515). This is the very basis of activity that Dewey advocated for meaningful education. The value of such activity is not based upon how accurately the scientific explanations put forth by the students match those accepted explanations within textbooks, but instead upon the ability of the students' explanations to resolve their present difficulties (LW 13: 232). As their educations continue, students continue to put forth, examine, negate, improve upon, and utilize their scientific explanations created from their own inquiries. If sustained, this type of activity becomes that of the philosopher-scientist, even within elementary school. 
As demonstrated above, Dewey's conceptions of education and experience entail the philosophical components of analysis, synthesis, and reconstructive thinking. This is especially true with regard to his conceptions of scientific education. One of the greatest tools Dewey offers for students (and teachers) in the context of science education is scientific explanation understood as a process in which the students engage rather than a product that the teachers are to deliver. By understanding and pursuing Dewey's conception of scientific explanation and how it can be utilized within science education, we come to a greater understanding of how students can engage in the experience of being philosopher-scientists.

Ultimately, the child as philosopher-scientist develops the skills of critical thought-such as reflection, abstraction, question generation, hypothesis formation, analogy - that provide a philosophical foundation for engaging in science as well as democratic citizenry. In addition, the child as philosopher-scientist begins to learn and implement the process of argumentation, which is a key component to generating and utilizing scientific explanations for solving problems. This is in stark contrast to the child who is not provided with the opportunity to be philosophical, or, worse, is trained to be nonphilosophical while engaging in scientific investigation. A nonphilosophical approach to science consists in the mere rote memorization of data and methods involving little to no critical inquiry. The student who is denied philosophical thought has fewer tools to develop, aside from that of passive memory and other unreflective processes. The philosopher-scientist develops a foundation in the same core of information, and, more importantly, a critical disposition toward multiple subjects that contributes to the development of higher-order thinking and processes of thought that creatively and actively contribute to solving problems and a life well lived. By fostering the child as philosopher-scientist, we thereby facilitate the development of thoughtful members of a larger democratic community of inquirers who are equipped to adapt to the ever-changing environment of which they are a part.

\section{Notes}

1. For an interesting collection of quotations from Dewey concerning his philosophy of science in science education, see Ansbacher.

2. This aspect of student-guided questioning is addressed in more depth with regard to mathematics education by Nadia Kennedy in this volume.

3. Regarding the ability for students to construct memories linked to positive emotions, see LaBar and Phelps.

\section{References}

Ansbacher, Ted. "An Interview with John Dewey on Science Education." The Physics Teacher 38 (April 2000): 224-27.

Barell, John. Developing More Curious Minds. Alexandria, VA: ASCD, 2003. 
Brookfield, Stephen D. and Preskill, S. Discussion as a Way of Teaching: Tools and Techniques for Democratic Classrooms. $2^{\text {nd }}$. ed. San Francisco, CA: Jossey-Bass, 2005.

Dewey, John. The Collected Works of John Dewey, 1882 - 1953. Edited by Jo Ann Boydston. Carbondale, IL: Southern Illinois University Press, 1969-1991.

Dewey, John. The Collected Works of John Dewey, 1882 - 1953: The Electronic Edition.

Edited by Larry A. Hickman. Charlottesville, VA: InteLex Corporation, 1996.

Duschl, Richaed A. Restructuring Science Education: The Importance of Theories and their Development. New York: Teachers College Press, 1990.

Getz, Lowell. “Fun in Research.” The Biologist 56 (1974): 86-89.

Kennedy, Nadia Stoyanova. "Lipman, Dewey, and Philosophical Inquiry in the Mathematics Classroom." Education and Culture 28, no. 2 (2012), 81-94.

Kyle Jr., William C., R. J. Bonnstetter, and T. Gadsen Jr. "An Implementation Study: An Analysis of Elementary Students' and Teachers' Attitudes toward Science in Process-Approach vs. Traditional Science Classes." Journal of Research in Science Teaching 25, no. 2 (1988): 103-20.

LaBar, K. S. and E. A. Phelps. "Arousal-Mediated Memory Consolidation: Role of the Medial Temporal Lobe in Humans." Psychological Science 9 (1998): 490-93. Lave, Jean. Cognition in Practice: Mind, Mathematics, and Culture in Everyday Life. Cambridge: Cambridge University Press, 1988.

Pollak, Victor L. "Science Education-I: The Spirit of Science." Journal of Science Education and Technology 2, no. 4 (1993): 513-19.

Reiser, Brian J., L. K. Berland, and L. Kenyon,. "Engaging Students in the Scientific Practices of Explanation and Argumentation: Understanding A Framework for K-12 Science Education." Science and Children (April 2012): 8-13.

Sandoval, William A. and Reiser, B. J. "Explanation-Driven Inquiry: Integrating Conceptual and Epistemic Scaffolds for Scientific Inquiry." Science Education 88 (2004): 345-72.

Sousa, David A. How the Brain Learns, Fourth Edition. Thousand Oaks, CA: Corwin, 2011.

Mark D. Tschaepe is Assistant Professor of Philosophy at Prairie View A\&M University.

E-mail: MDTschaepe@pvamu.edu 